Research Article

\title{
Overexpression of $\boldsymbol{m} \boldsymbol{f p A}$ Gene Increases Ciprofloxacin Resistance in Mycobacterium smegmatis
}

\author{
Aura Falco $\mathbb{D}^{1,2}$ Carlos Aranaga $\mathbb{D}^{1,3}$ Ivan Ocampo $\mathbb{D}^{1},{ }^{1}$ and Howard Takiff $\mathbb{D}^{2,4,5}$ \\ ${ }^{1}$ Grupo de Investigación en Microbiología, Industria y Ambiente (GIMIA), Facultad de Ciencias Básicas, \\ Universidad Santiago de Cali, Cali, Colombia \\ ${ }^{2}$ Laboratorio de Genética Molecular, Centro de Microbiología y Biología Celular, \\ Instituto Venezolano de Investigaciones Científicas, Km. 11, Carretera Panamericana, Caracas, Venezuela \\ ${ }^{3}$ Grupo de Investigación en Química y Biotecnología (QUIBIO), Facultad de Ciencias Básicas, Universidad Santiago de Cali, \\ Cali, Colombia \\ ${ }^{4}$ Integrated Mycobacterial Pathogenomics, Institut Pasteur, Paris, France \\ ${ }^{5}$ Department of Tuberculosis Control and Prevention, Shenzhen Nanshan Centre for Chronic Disease Control, Shenzhen, China \\ Correspondence should be addressed to Aura Falco; aura.falco00@usc.edu.co
}

Received 8 October 2020; Revised 5 February 2021; Accepted 3 March 2021; Published 22 March 2021

Academic Editor: Simona Nardoni

Copyright (c) 2021 Aura Falco et al. This is an open access article distributed under the Creative Commons Attribution License, which permits unrestricted use, distribution, and reproduction in any medium, provided the original work is properly cited.

Fluoroquinolones (FQs) are antibiotics useful in the treatment of drug-resistant tuberculosis, but FQ-resistant mutants can be selected rapidly. Although mutations in the DNA gyrase are the principal cause of this resistance, pentapeptide proteins have been found to confer low-level FQ resistance in Gram-negative bacteria. MfpA is a pentapeptide repeat protein conserved in mycobacterial chromosomes, where it is adjacent to a group of four highly conserved genes termed a conservon. We wished to characterize the transcriptional regulation of the $m f p A$ gene and relate its expression to ciprofloxacin resistance in $M$. smegmatis. Reverse transcription PCR showed that $m f p A$ gene is part of an operon containing the conservon genes. Using a transcriptional fusion, we showed that a promoter was located $5^{\prime}$ to the $m f p E A$ operon. We determined the promoter activity under different growth conditions and found that the expression of the operon increases slightly in late growth phases in basic $\mathrm{pH}$ and in subinhibitory concentrations of ciprofloxacin. Finally, by cloning the $m f p$ A gene in an inducible vector, we showed that induced expression of $m f p A$ increases the ciprofloxacin Minimal Inhibitory Concentration. These results confirm that increased expression of the $m f p \mathrm{~A}$ gene, which is part of the $m f p \mathrm{EA}$ operon, increases ciprofloxacin resistance in M. smegmatis.

\section{Introduction}

Fluoroquinolones (FQs) are the most important antibiotics in drug regimens used for treating Multidrug-Resistant Tuberculosis (MDR-TB) [1]. They exert a powerful bactericidal activity and can penetrate macrophages, where the tuberculosis-causing Mycobacterium tuberculosis bacilli reside [2]. There are, however, reservations regarding FQ use because resistant mutants can be selected in a remarkably short time [1]. The targets of the FQs are the DNA gyrase and DNA Topoisomerase IV. Mycobacteria only contain the gyrase, which catalyses negative supercoiling of bacterial DNA [2], and most FQ resistance is caused by substitutions in a few critical amino acids located in the Quinolone
Resistance-Determining Regions (QRDR) of the GyrA and GyrB subunits, although efflux pumps have also been implicated in the development of resistance [3-5].

Another novel mechanism of FQ resistance involves proteins belonging to the Pentapeptide Repeat Family (PRF). In Gram-negative bacteria, the genes of the qnr family encode pentapeptide proteins that are generally found on transmissible plasmids and confer low-level FQ resistance [6-9]. The proteins of the pentapeptide family are composed almost entirely of a repeating five amino acid motif, in which every fifth amino acid is either leucine or phenylalanine [10]. Pentapeptides were initially associated with FQ resistance when it was found that a plasmid containing the $m f p A$ (Mycobacterial Fluoroquinolone Resistance Protein) gene of 
Mycobacterium smegmatis increased the MICs to ciprofloxacin and sparfloxacin in this bacterium. This gene encodes a protein of 192 amino acids with 32 pentapeptide repeats in tandem [11]. In M. tuberculosis, the gene $R v 3361 \mathrm{C}$ encodes a pentapeptide protein (termed MtMfpA) of 183 amino acids that has $67 \%$ amino acid identity with the M. smegmatis MfpA. The structure of the MtMfpA protein is a right-handed helix with the size, shape, and charge distribution reminiscent of $\mathrm{B}$-form DNA, and it has been suggested that MfpA may compete with DNA for binding to the DNA gyrase. Because the FQs only bind to the gyrase when it is complexed with DNA, the binding of MfpA to the gyrase could either prevent the formation of the gyrase DNA complex, or replace DNA in a FQ-inhibited complex, thereby releasing the gyrase from FQ inhibition, and thus conferring resistance [12].

Very conserved $m f p A$ genes are found on the chromosomes of all known mycobacterial genomes [10]. Their biological function is unknown, but they are accompanied at their $5^{\prime}$-end by a group of four highly conserved genes, termed a conservon, which have also been found in several other Actinobacteria, the class of bacteria to which mycobacteria belong. Conservons appear to be regulatory units that respond to unknown signals, and the fifth gene in the unit, where $m f p A$ is found, is different in other Actinomycetes [13].

Because the FQs are an important drug for curing patients with MDR-TB $[14,15]$, we attempted to clarify the role of pentapeptide proteins in FQ resistance through genetic characterization of the $m f p$ A gene in $M$. smegmatis. We found that the $m f p \mathrm{~A}$ gene is part of a transcriptional unit with the four upstream conservon genes. Moreover, we showed that the promoter region for this operon, termed $m f p$ EA, was at $5^{\prime}$-end of the $m f p$ E gene. Finally, the $m f p$ A gene was cloned into an inducible vector to increase its expression and thereby confirm that increased expression leads to increased FQ resistance. The results suggest a possible link between MfpA and FQ resistance in $M$. smegmatis, which could have important implications for FQ resistance in $M$. tuberculosis.

\section{Materials and Methods}

\subsection{Bacterial Strains and Plasmids}

Escherichia coli XL1-Blue, used for cloning, was grown at $37^{\circ} \mathrm{C}$ in Luria-Bertani (LB) broth or on LB agar (Difco ${ }^{\mathrm{TM}}$, Cat. No. 244520, USA). Mycobacterium smegmatis strains were grown at $37^{\circ} \mathrm{C}$ in Middlebrook $7 \mathrm{H} 9$ broth (Difco ${ }^{\mathrm{TM}}$, Cat. No. 271310, USA) or 7H10 agar (Difco ${ }^{\mathrm{TM}}$, Cat. No. 262710, USA) supplemented with Tween (0.05\%), glycerol $(0.2 \%)$, and $\mathrm{OAD}(10 \%)$. The plasmids and bacterial strains used in this study are described in Table 1.

2.2. DNA Manipulation. The genomic DNA of M. smegmatis was isolated as described by van Soolingen et al. [19]. Escherichia coli was electroporated according to
Hanahan et al. [20], while M. smegmatis was electroporated following the protocol of Jacobs et al. [21].

2.3. Cloning. A transcriptional fusion between the putative promoter region of the $m f p \mathrm{EA}$ operon and the lacZ gene in pJEM15 was constructed by PCR amplifying a 326 bp fragment at the $5^{\prime}$-end of the $m f p E$ gene with primers $\mathrm{mfp} 5$ and mfp11 (Figure 1, Tables 1 and 2) and inserting it into the $K p n I$ restriction site on plasmid pJEM15. The $m f p A$ gene was inserted into the expression vector pALACE by amplifying with primers mfp16 and mfp17 (Figure 1, Tables 1 and 2) and digesting with $\mathrm{Bam} \mathrm{HI}$ and $\mathrm{ClaI}$. The resulting constructs were verified by sequencing (Macrogen, South Korea) using the corresponding primers (Table 2).

2.4. Determination of Promoter Activity. The promoter activity of the transcriptional fusion was evaluated by the detection of beta-galactosidase activity in $7 \mathrm{H} 9$ media with Tween $(0.05 \%)$, glycerol (0.2\%), and OAD (10\%). Mycobacterium smegmatis $\mathrm{mc}^{2} 155$ was electroporated with plasmids pJEM15-5.11 and vector pJEM15 (Table 1). The promoter activity was determined by detecting Relative Fluorescence Units (RFU) produced by hydrolysis of the C2FDG (5-acetylamino di-beta-D-galactopyranoside) substrate (Molecular Probes, OR, USA). The assay was initiated by inoculating each strain into $5 \mathrm{ml}$ of $7 \mathrm{H} 9$ supplemented with kanamycin $(25 \mu \mathrm{g} / \mathrm{ml})$ and incubating at $37^{\circ} \mathrm{C}$ with agitation for 3 days. Subsequently, $0.1 \mathrm{ml}$ of these cultures was inoculated into $10 \mathrm{ml}$ of $7 \mathrm{H} 9$ with kanamycin $(25 \mu \mathrm{g} / \mathrm{ml})$ and incubated at $37^{\circ} \mathrm{C}$ with agitation until reaching $\mathrm{OD}_{600 \mathrm{~nm}}$ 0.5 . Fluorescence assays were performed in 96-well microtiter plates using $90 \mu \mathrm{l}(\sim 0.5 \times 104$ cells/well $)$ of each culture (previously diluted $1: 10^{4}$ ) mixed with $10 \mu \mathrm{l}$ of the C2FDG fluorophore $(33 \mu \mathrm{M})$. Bacteria were incubated with this substrate at $37^{\circ} \mathrm{C}$ for 96 hours, during which $\beta$-galactosidase activity was detected by exciting at a wavelength of $485 \pm 20$ $\mathrm{nm}$ and measuring emission at $530 \pm 25$, using a SpectraMax $^{\circledR}$ Gemini XS instrument (Molecular Devices, CA, USA) [22]. In parallel, mycobacterial growth was followed measuring $\mathrm{OD}_{600 \mathrm{~nm}}$ as a function of time. These assays were done in triplicate, and the results show the standard deviation for each measurement.

\subsection{Evaluation of Promoter Activity in Different Growth} Conditions. The promoter activity of $m f p$ EA operon under different conditions was evaluated as described above, with some modifications. Mycobacterium smegmatis $\mathrm{mc}^{2} 155$ was transformed with plasmid pJEM15-5.11 and vector pJEM15 (Table 1) and grown for 96 hours in $7 \mathrm{H} 9$ in different conditions: $7 \mathrm{H} 9$ supplemented with ciprofloxacin $(0.125 \mu \mathrm{g} /$ $\mathrm{ml}$ ) and $7 \mathrm{H} 9$ adjusted to $\mathrm{pH} 8.0$ by the addition of concentrated $\mathrm{NaOH}$ and then filter sterilized through a disposable polyethersulfone filter with a $0.45 \mathrm{~mm}$ pore size [23]. These assays were done in triplicate, and the results show the standard deviation for each measurement. 
TABLE 1: Strains and plasmids used in this work.

\begin{tabular}{|c|c|c|}
\hline Strains and plasmids & Relevant characteristics & References \\
\hline E. coli XL1-Blue & $\begin{array}{l}\text { recA, endA, gyrA, thi-1, hsd } \mathrm{R} 17, \sup \mathrm{E} 44, \operatorname{rel} \mathrm{A}, \text { lac }\left[\mathrm{F}^{\prime} \text { pro } \mathrm{AB} \text { lac } \mathrm{I}^{\mathrm{q}} \mathrm{Z} \Delta \mathrm{M} 15 \operatorname{Tn} 10\right. \\
\left.\left(\text { Tet }^{\mathrm{R}}\right)\right] .\end{array}$ & Stratagene, CA, USA \\
\hline M. smegmatis $\mathrm{mc}^{2} 155$ & Mutant, transformable strain of $M$. smegmatis derived from the strain $\mathrm{mc}^{2} 6$. & Snapper et al. [16] \\
\hline $\begin{array}{l}\text { M. smegmatis } m c^{2} 155 \text { gyrA } \\
\text { D94G }\end{array}$ & $\begin{array}{c}\text { FQ-resistant mutant strain of } \begin{array}{c}\text { M. smegmatis } \mathrm{mc}^{2} 155 \text { with the gyrA D94G } \\
\text { substitution. }\end{array}\end{array}$ & Our laboratory \\
\hline pJEM15 & $\begin{array}{l}\text { Cloning and shuttle vector }(9.5 \mathrm{kbp}) \text { with a promoterless lacZ gene. } \\
\qquad \operatorname{Kan}^{\mathrm{R}}(25 \mu \mathrm{g} / \mathrm{ml}) .\end{array}$ & Timm et al., [17] \\
\hline pJEM15-5.11 & pJEM15 cloning vector with the putative promoter region of the $m f p$ EA operon. & Thi \\
\hline pALACE & $\begin{array}{l}\text { Expression and shuttle vector ( } 8221 \mathrm{bp}) \text { with a strong promoter (Pace) induced with } \\
\text { acetamide. } \operatorname{Hyg}^{\mathrm{R}}(50 \mu \mathrm{g} / \mathrm{ml}) .\end{array}$ & $\begin{array}{l}\text { Lakshminarayan et al. } \\
{[18]}\end{array}$ \\
\hline pALACE- $m f p A$ & pALACE vector with the $m f p A$ gene of $M$. smegmatis. & This study \\
\hline
\end{tabular}

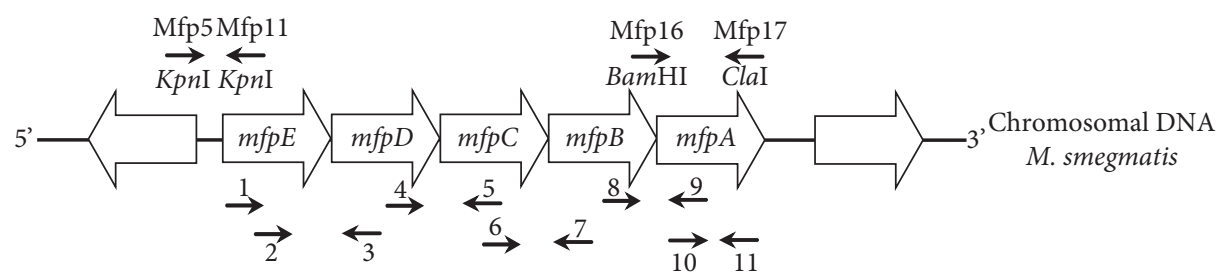

Figure 1: Schematic representation of the $m f p$ EA operon. The arrows indicate the regions to be amplified with different primers.

TABle 2: Primers used in this study.

\begin{tabular}{lc}
\hline $\begin{array}{c}\text { Primer } \\
\text { name }\end{array}$ & Primer sequence \\
\hline mfp5 & TTTTTTGGTACCTACCGGTATTCGGCGCGAT \\
mfp11* & TTTTTTGGTACCCGCCCAACCGTGCCATCG \\
mfp16* & TTTTTTGGATCGCTGCCCGGCTGAGGCTT \\
mfp17* & TTTTTTATCGATCTGCTCGCGGTGAGAAAC \\
Random & $\left(\mathrm{d}(\mathrm{N})_{9}\right.$ where $\left.\mathrm{N}=\mathrm{A}, \mathrm{C}, \mathrm{G}, \mathrm{T}\right)$ \\
primers 9 & TTGCAGCAGCGGGTGGATTC \\
1 & GACGAGGATCTGGAGCCGGCAT \\
10 & GAGCGTGAAGTCGCACTCGA \\
11 & AAACGACAACGGTGCCGAGG \\
2 & GCGTTCGACCGGCATGTTCT \\
3 & GGCTACGTGCTGCAATCGGT \\
4 & GCGCGTTGGGTGTGTCGAGA \\
5 & GCGTGGCGAGAGTGCTGATC \\
6 & GTGGTGGTGCGTTTGTCGGG \\
7 & GCCAAAGCATCCCACGCACG \\
8 & CGTGCTGTGCCAGATGGTGC \\
9 & CCAGCAGCCGCGGTAATACG \\
$16 S$ forward & ATCGG(C/T)TACCTTGTTACGACTTC \\
16 reverse &
\end{tabular}

${ }^{*}$ Underlined letters indicate restriction sites. Figure 1 shows target sites of primers in $m f p$ EA operon.

2.6. Inducible Expression of MfpA. MfpA expression was induced according to Lakshminarayan et al. [18]. In brief, the $M$. smegmatis strains used in this assay $\left(\mathrm{mc}^{2} 155\right.$ and $\mathrm{mc}^{2} 155$ gyrA, see Table 1) were electroporated with pALACE or pALACE- $m f p$ A (Table 2) and grown overnight in 7H9 supplemented with $1 \%$ glucose. They were then washed once in 7H9 broth without glucose and resuspended to an $\mathrm{OD}_{600 \mathrm{~nm}} 1.0 \mathrm{in} 7 \mathrm{H} 9$ medium lacking glucose. Induction was initiated by adding acetamide to a final concentration of $0.02 \%$ and incubating overnight with constant agitation.
Controls without acetamide were set up in parallel [18]. Subsequently, $20 \mu \mathrm{l}$ of $10^{-4}$ and $10^{-5}$ dilutions were plated on $7 \mathrm{H} 10$ supplemented with hygromycin $(50 \mu \mathrm{g} / \mathrm{ml})$, acetamide $(0.02 \%)$, and increasing concentrations of ciprofloxacin $(0$, $0.25,0.5,1,2,4,8,16$, and $32 \mathrm{y} 64 \mu \mathrm{g} / \mathrm{ml})$. These assays were done in triplicate. We used as positive and negative controls the antibiotics hygromycin $(50 \mu \mathrm{g} / \mathrm{ml})$ and kanamycin $(25 \mu \mathrm{g} / \mathrm{ml})$, respectively.

2.7. RNA Extraction and Reverse Transcription PCR. Total RNA from $M$. smegmatis was isolated as previously described [24], from bacteria grown to $\mathrm{OD}_{600 \mathrm{~nm}} 0.5$. Residual DNA in the mycobacterial total RNA preparations was removed using DNase I according to the manufacturer's instructions (Sigma-Aldrich, Inc., Darmstadt, Germany). A control with no reverse transcriptase (NRT) was used for a PCR reaction with primers to $16 \mathrm{~S}$ rRNA [25] (Table 2) to confirm complete DNA removal. The M-MuLV Reverse Transcriptase (New England Biolabs, MA, USA) and random primers 9 (New England Biolabs, MA, USA) (Table 2) were used to produce cDNA according to the manufacturer's instructions. The cDNA was used to amplify the $m f p$ genes, in triplicate, using ThermoPol ${ }^{\circledR}$ (New England Biolabs, MA, USA) and specific primers (see Figure 1 and Table 1: $m f p E D$, $m f p$ DC, $m f p \mathrm{CB}, m f p \mathrm{BA}$, and $m f p \mathrm{~A}$ ). After PCR amplification, the fragments were sequenced with the amplification primers in the forward and reverse directions (Macrogen, Korea).

\section{Results}

3.1. Identification of the mfpEA Operon in Mycobacterium smegmatis. We first used RT-PCR to determine whether $m f p A$ is part of a transcriptional unit with the four upstream 


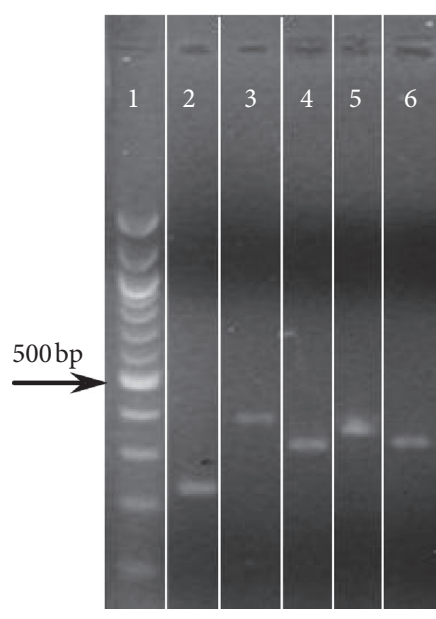

(a)

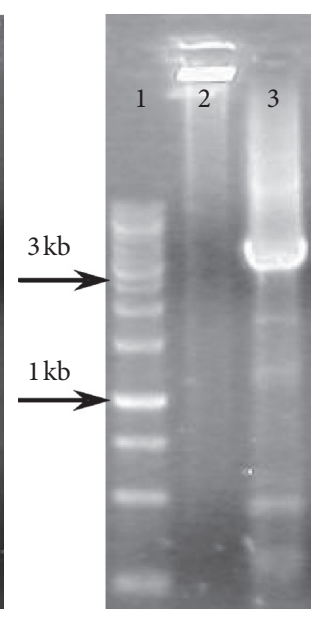

(b)
FIgURE 2: RT-PCR of the $m f p$ EA operon. (a) Lane 1: MWM $100 \mathrm{bp}$ ladder (NEB); Lane 2: RT-PCR using primers 10-11; Lane 3: RTPCR using primers 8-9; Lane 4: RT-PCR using primers 6-7; Lane 5: RT-PCR using the primers 4-5; and Lane 6: RT-PCR using primers 2-3. (b) Lane 1: MWM O' Gene Ruler $1 \mathrm{~kb}$ DNA ladder (Thermo Scientific); Lane 2: No Reverse Transcriptase control (NRT) using primers 1-11; and Lane 3: RT-PCR using primers 1-11.

conservon genes $(m f p \mathrm{E}, m f p \mathrm{D}, m f p \mathrm{C}$, and $m f p \mathrm{~B}$. See Figure 1 and Table 2). The amplification yielded a $221 \mathrm{bp}$ fragment of the $m f p$ A gene (Figure 2(a)), as well as fragments corresponding to the regions between $m f p \mathrm{E}$ and $m f p \mathrm{~A}$ (4712 bp, see Figure 2(b)), $m f p \mathrm{E}$ and $m f p \mathrm{D}(312 \mathrm{bp}), m f p \mathrm{D}$ and $m f p \mathrm{C}$ $(341 \mathrm{pb}), m f p \mathrm{C}$ and $m f p \mathrm{~B}(311 \mathrm{bp})$, and $m f p \mathrm{~B}$ and $m f p \mathrm{~A}$ (375 $\mathrm{bp)}$ (Figure 2(a)), indicating that the five genes belong to the same transcriptional unit, which we term the $m f p$ EA operon.

3.2. Promoter Activity for the mfpEA Operon. A $326 \mathrm{bp}$ fragment including the $5^{\prime}$-end of the $m f p \mathrm{E}$ gene was cloned in front of the promoterless lacZ gene in vector pJEM15 to obtain the transcriptional fusion plasmid pJEM5-11 (Figure 1, Table 1). Subsequently, M. smegmatis mc $^{2} 155$ strains containing pJEM15 (control) or pJEM-5-11 (Table 1) were grown in $7 \mathrm{H} 9$ broth with $\mathrm{C} 2 \mathrm{FDG}$ substrate $(0.33 \mu \mathrm{M})$, and promoter activity was measured as fluorescence resulting from the hydrolysis of C2FDG by beta-galactosidase. The fluorescence produced with plasmid pJEM-5-11 (grey dots) indicated that the cloned fragment contained a promoter whose activity correlated with bacterial growth (black dots) (Figure 3).

The promoter activity was also evaluated in different growth conditions (see Materials and Methods). A slightly increased expression was seen in $7 \mathrm{H} 9$ growth media at $\mathrm{pH}$ 8.0 (Figure $4(\mathrm{a})$ ) and with a subinhibitory concentration of ciprofloxacin $(0.125 \mu \mathrm{g} / \mathrm{ml}$ ) (Figure $4(\mathrm{~b}))$, but only after 96 hours of growth.

3.3. Determination of the Relation between $m f p A$ and Ciprofloxacin Resistance. To determine the relation between $m f p A$ and ciprofloxacin resistance, the $m f p A$ gene was cloned into the pALACE vector to create plasmid pALACE-

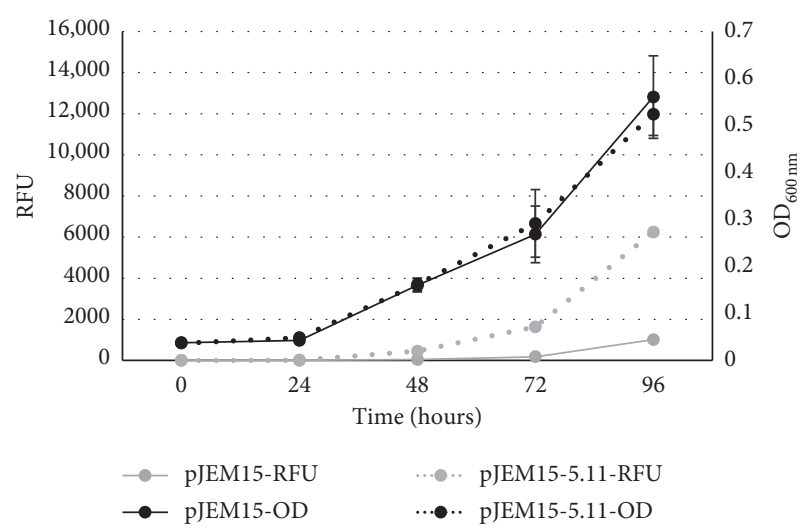

Figure 3: Promoter activity of the putative $m f p$ EA operon. Curves labelled OD represent mycobacterial growth while the RFU curves indicate beta-galactosidase activity.

$m f p \mathrm{~A}$ (Figure 1, Tables 1 and 2), in which the $m f p \mathrm{~A}$ is expressed from an acetamide inducible promoter. The MICs for ciprofloxacin were compared between two strains of $M$. smegmatis containing either the empty vector or pALACE$m f p A$ : wild type (mc $\left.{ }^{2} 155\right)$ and $\mathrm{mc}^{2} 155$ gyrA D94G (Table 2). The induction of $m f p A$ expression with acetamide produced increased ciprofloxacin resistance in both $M$. smegmatis strains. In the wild-type strain, the resistance to this antibiotic increased fourfold, while in the strain with the mutation in gyrA gene, it increased twofold (Table 3).

The relative changes in $\mathrm{MIC}^{*}$ were expressed through the relationship (2)/(1).

\section{Discussion}

We have shown that the gene encoding the pentapeptide $m f p \mathrm{~A}$ is the fifth gene in a transcriptional unit that also contains the four genes of the conservon, $m f p \mathrm{EDCB}$. We have termed this the $m f p$ EA operon. We also confirmed that increased transcription of $m f p \mathrm{~A}$ increases FQ resistance, even in a strain containing a FQ resistance-conferring gyr $\mathrm{A}$ mutation. Although conservons are also found in several other actinobacteria, only in mycobacteria are they located $5^{\prime}$ to a pentapeptide encoding gene [10]. Judging by the domains identified in the four conservon encoded proteins, the unit appears to have a regulatory function: $m f p \mathrm{~B}$ is similar to a signal recognition particle receptor beta subunit of a GTPase; $m f p C$ contains a helix-turn helix motif; $m f p \mathrm{D}$ is similar to regulators with Ras-like GTPase activity; and $m f p \mathrm{E}$ is similar to signal transduction histidine kinases [13, 26, 27].

The fluoroquinolones act by inhibiting the activity of the gyrase, freezing the DNA gyrase complex with doublestranded breaks in the DNA that cannot be relegated. With the acetamide-induced promoter, we showed that increasing $m f p \mathrm{~A}$ expression increases the FQ MICs, perhaps, as has been proposed, by mimicking and replacing the chromosomal DNA, thereby releasing the DNA trapped in the gyrase-FQ complex [10, 28, 29]. $m f p A$ has been shown to interact with the DNA gyrase, an essential ATPase enzyme that introduces negative supercoils into the DNA molecule. Because mycobacteria contain no Topoisomerase IV, the 


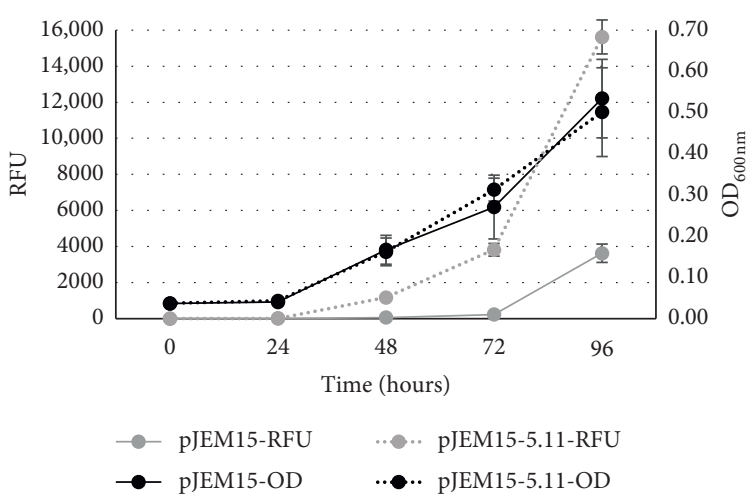

(a)

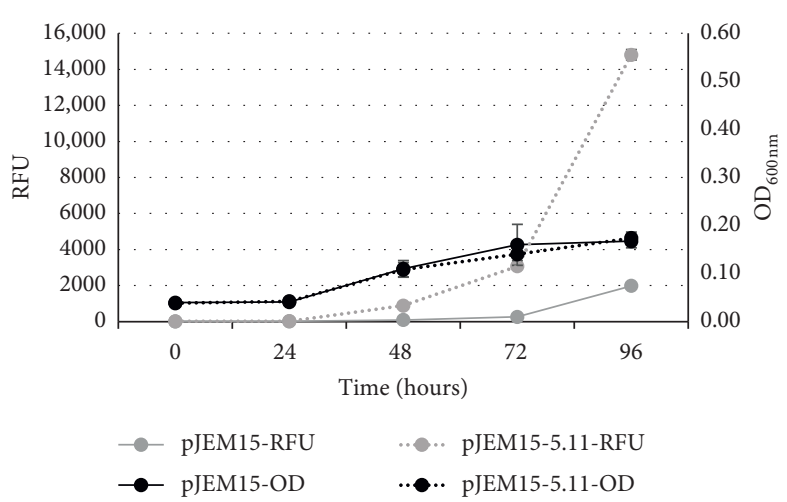

(b)

FIgURE 4: Promoter activity of the putative operon $m f p$ EA operon in different growth conditions. (a) $7 \mathrm{H} 9$ (pH 8.0). (b) $7 \mathrm{H} 9$ with ciprofloxacin $(0.125 \mu \mathrm{g} / \mathrm{ml})$. Curves labelled OD represent mycobacterial growth, while the RFU curves indicate beta-galactosidase activity.

TABle 3: Effect of $m f p$ A overexpression on ciprofloxacin MIC.

\begin{tabular}{lccc}
\hline \multirow{2}{*}{ Strains } & \multicolumn{2}{c}{ MIC $(\mu \mathrm{g} / \mathrm{ml})$ ciprofloxacin } & Relative change in the MIC* \\
& pALACE $(1)$ & pALACE- $m f p A(2)$ & 4 \\
$\mathrm{mc}^{2} 155$ & 0.25 & 1 & 2 \\
$\mathrm{mc}^{2} 155$ gyrA D94G & 1 & 2 & 4 \\
\hline
\end{tabular}

gyrase presumably also performs its function of decatenating the two interlocking circular chromosomes resulting from DNA replication. Because it must perform these two functions, one of which is linked to DNA replication, it is possible that either the expression or the activity of $\mathrm{MfpA}$ and its interaction with the gyrase could be regulated by the conservon unit, but none of the conditions we tested appeared to substantially increase the transcription of the $m f p$ EA operon. We reasoned that inhibition of the gyrase with ciprofloxacin might lead to increased transcription of the $m f p$ EA operon to mitigate the effects of the drug, but we found just a slight increase in promoter activity only after 96 hours of culture. Other studies have shown that the expression of $q n r$ genes increases with the SOS response [30], and the FQ's have been shown to induce the SOS response [31], but the increased promoter activity after 96 hours does not appear typical of an SOS response. We tentatively conclude that if the conservon somehow regulates $m f p A$ activity, the regulation is not at the level of transcription. It is possible that the $m f p$ EA operon has additional, internal promoters that are regulated, or the conservon acts at a posttranscriptional level by somehow modulating MfpA function through an alteration of GTPase or ATPase activity suggested by the conserved domains in the putative proteins encoded by the conservon genes $m f p \mathrm{~B}$ and $m f p \mathrm{D}$, respectively.

With the lac Z fusion, we showed that there was a promoter upstream of $m f p E$ that expresses the whole operon, including $m f p \mathrm{~A}$, but promoter motifs are heterogeneous in mycobacteria and we could not identify the sequence of the putative promoter [32]. However, any mutation that increases promoter activity would raise $m f p \mathrm{~A}$ expression and thereby increase FQ resistance. In $M$. tuberculosis, the arrangement of the $m f p$ EDCBA genes is the same as in M. smegmatis, and mfpA has been shown to increase FQ resistance in $M$. tuberculosis family member $M$. bovis BCG [11]. mfpA could therefore contribute to intrinsic levels of resistance to the FQs in M. tuberculosis [3], and any mutation in the $m f p$ EA promoter that increases its expression would be expected to increase FQ resistance. If these mutations occur in strains with FQ resistance mutations in the gyrase, they might increase the MIC in an additive manner. We found that induced expression of $m f p A$ doubled the ciprofloxacin MIC in a strain containing the gyrA D94G mutation, a high-level resistance mutation frequently found in FQ resistant strains of $M$. tuberculosis. Alternatively, if a mutation augmenting the expression of $m f p$ A occurs before QRDR mutations, it could increase the tolerance to low levels of FQs and thereby increase the frequency of high-level resistance gyrase mutations. To our knowledge, however, mutations in the $m f p$ EA operon have not been described in drug-resistant M. tuberculosis, although it is not clear that this operon has been considered a site deserving careful examination.

\section{Conclusions}

The $m f p$ A gene, encoding the pentapeptide MfpA, is the fifth gene in the $m f p \mathrm{EA}$ operon that contains the four genes of the conservon, $m f p \mathrm{EDCB}$, and is expressed from a promoter located $5^{\prime}$ to $m f p \mathrm{E}$. Overexpression of the $m f p \mathrm{~A}$ gene increases ciprofloxacin resistance in $M$. smegmatis, even in strains with high-level FQ-resistance gyrA mutations.

\section{Data Availability}

The data used to support the findings of this study are included within the article and are available from the corresponding author upon request. 


\section{Conflicts of Interest}

The authors declare that there are no conflicts of interest regarding the publication of this paper.

\section{Acknowledgments}

The authors would like to express their gratitude to Dr. Carlos Sanz for checking the English language of the manuscript. This research was funded by Dirección General de Investigaciones of Universidad Santiago de Cali under call no. 01-2021, the Potts Memorial Foundation, and Instituto Venezolano de Investigaciones Científicas (IVIC).

\section{References}

[1] H. Takiff and E. Guerrero, "Current prospects for the fluoroquinolones as first-line tuberculosis therapy," Antimicrobial Agents and Chemotherapy, vol. 55, no. 12, pp. 5421-5429, 2011.

[2] G. Di Perri and S. Bonora, "Which agents should we use for the treatment of multidrug-resistant Mycobacterium tuberculosis?" Journal of Antimicrobial Chemotherapy, vol. 54, no. 3, pp. 593-602, 2004.

[3] C. Mayer and H. Takiff, "The molecular genetics of fluoroquinolone resistance in Mycobacterium tuberculosis," Microbiology Spectrum, vol. 2, no. 4, pp. 1-22, 2014.

[4] H. E. Takiff, L. Salazar, C. Guerrero et al., "Cloning and nucleotide sequence of Mycobacterium tuberculosis gyrA and gyrB genes and detection of quinolone resistance mutations," Antimicrobial Agents and Chemotherapy, vol. 38, no. 4, pp. 773-780, 1994.

[5] A. S. Ginsburg, J. H. Grosset, and W. R. Bishai, "Fluoroquinolones, tuberculosis, and resistance," The Lancet Infectious Diseases, vol. 3, no. 7, pp. 432-442, 2003.

[6] S. S. Hegde, M. W. Vetting, L. A. Mitchenall, A. Maxwell, and J. S. Blanchard, "Structural and biochemical analysis of the pentapeptide repeat protein $E f s \mathrm{Qnr}$, a potent DNA gyrase inhibitor," Antimicrobial Agents and Chemotherapy, vol. 55, no. 1, pp. 110-117, 2011.

[7] M. W. Vetting, S. S. Hegde, Y. Zhang, and J. S. Blanchard, "Pentapeptide-repeat proteins that act as topoisomerase poison resistance factors have a common dimer interface," Acta Crystallographica Section F Structural Biology and Crystallization Communications, vol. 67, no. 3, pp. 296-302, 2011.

[8] M. W. Vetting, S. S. Hegde, M. Wang, G. A. Jacoby, D. C. Hooper, and J. S. Blanchard, "Structure of QnrB1, a plasmid-mediated fluoroquinolone resistance factor," Journal of Biological Chemistry, vol. 286, no. 28, pp. 25265-25273, 2011.

[9] G. A. Jacoby and D. C. Hooper, "Phylogenetic analysis of chromosomally determined Qnr and related proteins," $A n$ timicrobial Agents and Chemotherapy, vol. 57, no. 4, pp. 1930-1934, 2013.

[10] M. W. Vetting, S. S. Hegde, J. E. Fajardo et al., "Pentapeptide repeat proteins," Biochemistry, vol. 45, no. 1, pp. 1-10, 2006.

[11] C. Montero, G. Mateu, R. Rodriguez, and H. Takiff, "Intrinsic resistance of Mycobacterium smegmatis to fluoroquinolones may Be influenced by new pentapeptide protein MfpA," Antimicrobial Agents and Chemotherapy, vol. 45, no. 12, pp. 3387-3392, 2001.
[12] S. S. Hegde, M. W. Vetting, S. L. Roderick et al., "A fluoroquinolone resistance protein from Mycobacterium tuberculosis that mimics DNA," Science, vol. 308, no. 5727, pp. 1480-1483, 2005.

[13] M. Komatsu, H. Takano, T. Hiratsuka et al., "Proteins encoded by the conservon of Streptomyces coelicolor A3(2) comprise a membrane-associated heterocomplex that resembles eukaryotic G protein-coupled regulatory system," Molecular Microbiology, vol. 62, no. 6, pp. 1534-1546, 2006.

[14] M. R. Farhat, K. R. Jacobson, M. F. Franke, D. Kaur, M. Murray, and C. D. Mitnick, "Fluoroquinolone resistance mutation detection is equivalent to culture-based drug sensitivity testing for predicting multidrug-resistant tuberculosis treatment outcome: a retrospective cohort study," Clinical Infectious Diseases, vol. 65, no. 8, pp. 1364-1370, 2017.

[15] A. Trébucq, V. Schwoebel, Z. Kashongwe et al., "Treatment outcome with a short multidrug-resistant tuberculosis regimen in nine African countries," The International Journal of Tuberculosis and Lung Disease, vol. 22, no. 1, pp. 17-25, 2018.

[16] S. B. Snapper, R. E. Melton, S. Mustafa, T. Kieser, and W. R. J. Jacobs Jr., "Isolation and characterization of efficient plasmid transformation mutants of Mycobacterium smegmatis," Molecular Microbiology, vol. 4, no. 11, pp. 1911-1919, 1990.

[17] J. Timm, E. M. Lim, and B. Gicquel, "Escherichia colimycobacteria shuttle vectors for operon and gene fusions to lacZ: the pJEM series," Journal of Bacteriology, vol. 176, no. 21, pp. 6749-6753, 1994.

[18] H. Lakshminarayan, S. Narayanan, H. Bach, and K. G. P. Sundaram, Y. Av-Gay, Molecular cloning and biochemical characterization of a serine threonine protein kinase, PknL, from Mycobacterium tuberculosis," Protein Expression and Purification, vol. 58, no. 2, pp. 309-317, 2008.

[19] D. v. Soolingen, P. E. W. de Haas, P. W. M. Hermans, and J. D. A. Van Embden, "DNA Fingerprinting of mycobacterium tuberculosis," Methods in Enzymology, vol. 235, pp. 196-205, 1994.

[20] D. Hanahan, J. Jessee, and F. R. Bloom, "Plasmid transformation of Escherichia coli and other bacteria," Methods in Enzymology, vol. 204, pp. 63-113, 1991.

[21] W. R. Jacobs, G. V. Kalpana, J. D. Cirillo et al., "Genetic systems for mycobacteria," Bacterial Genetic System, vol. 204, pp. 537-555, 1991.

[22] B. Rowland, A. Purkayastha, C. Monserrat, Y. Casart, H. Takiff, and K. A. McDonough, "Fluorescence-based detection of lac $Z$ reporter gene expression in intact and viable bacteria including Mycobacterium species," FEMS Microbiology Letters, vol. 179, no. 2, pp. 317-325, 1999.

[23] L. Richter and B. Saviola, "The lipF promoter of Mycobacterium tuberculosis is upregulated specifically by acidic $\mathrm{pH}$ but not by other stress conditions," Microbiological Research, vol. 164, no. 2, pp. 228-232, 2009.

[24] T. R. Rustad, D. M. Roberts, R. P. Liao, and D. R. Sherman, "Isolation of mycobacterial RNA," in Mycobacteria Protocols. Methods in Molecular Biology (Methods and Protocols), T. Parish and A. Brown, Eds., vol. 465pp. 13-22, Springer, Manhattaan, NY, USA, 2nd edition, 2008.

[25] J.-J. Lu, C.-L. Perng, S.-Y. Lee, and C.-C. Wan, "Use of PCR with universal primers and restriction endonuclease digestions for detection and identification of common bacterial pathogens in cerebrospinal fluid," Journal of Clinical Microbiology, vol. 38, no. 6, pp. 2076-2080, 2000.

[26] S. C. G. Rison, S. L. Kendall, F. Movahedzadeh, and N. G. Stoker, "The mycobacterial two-component regulatory 
systems," in Mycobacterium Tuberculosis Molecular Microbiology, T. Parish, Ed., Horizon Bioscience, London, UK, 2005.

[27] S. D. Bentley, K. F. Chater, A.-M. Cerdeño-Tárraga et al., "Complete genome sequence of the model actinomycete Streptomyces coelicolor A3(2)," Nature, vol. 41, pp. 141-147, 2002.

[28] G. A. Jacoby, K. E. Walsh, D. M. Mills et al., "qnrB, another plasmid-mediated gene for quinolone resistance," Antimicrobial Agents and Chemotherapy, vol. 50, no. 4, pp. 1178$1182,2006$.

[29] A. Mérens, S. Matrat, A. Aubry et al., "The pentapeptide repeat proteins $\mathrm{Mfp}_{\mathrm{Mt}}$ and QnrB4 exhibit opposite effects on DNA gyrase catalytic reactions and on the ternary gyrase-DNAquinolone complex," Journal of Bacteriology, vol. 191, no. 5, pp. 1587-1594, 2009.

[30] R. Monárrez, Y. Wang, Y. Fu et al., "Genes and proteins involved in qnrS1 Induction," Antimicrobial Agents and Chemotherapy, vol. 62, no. 9, pp. 1-12, 2018.

[31] G. Yim, J. McClure, M. G. Surette, and J. E. Davies, "Modulation of Salmonella gene expression by subinhibitory concentrations of quinolones," The Journal of Antibiotics, vol. 64, no. 1, pp. 73-78, 2011.

[32] M. A. Mulder, H. Zappe, and L. M. Steyn, "Mycobacterial promoters," Tubercle and Lung Disease, vol. 78, no. 5-6, pp. 211-223, 1997. 